\title{
BRAQUIÓPODOS CHONETOIDEOS DEL CARBONÍFERO DE MÉXICO
}

\author{
Francisco SOUR TOVAR ${ }^{1}$ y M. Luisa MARTÍNEZ \\ CHACÓN ${ }^{2}$
}

\author{
${ }^{1}$ Museo de Paleontología, Facultad de Ciencias, Universidad Nacional Autó- \\ noma de México. Ciudad Universitaria, México D. F., 04510, México. \\ fst@hp.fciencias.unam.mx \\ ${ }^{2}$ Departamento de Geología, Universidad de Oviedo, Jesús Arias de Velasco \\ s/n, Oviedo 33005, España.mmchacon@geol.uniovi.es
}

Sour Tovar, F. y Martínez Chacón, M.L. 2004. Braquiópodos chonetoideos del Carbonífero de México. [Chonetoidea (Brachiopoda) from the Carboniferous of Mexico.] Revista Española de Paleontología, 19 (2), 125-138. ISSN 0213-6937.

\begin{abstract}
Four species of chonetids from the Carboniferous of Mexico are described. The anopliid Yagonia collinsoni Carter comes from the Vicente Guerrero Formation (Osagean, Lower Mississippian), Tamaulipas, in northeastern Mexico. The anopliid Tornquistia sp., and the rugosochonetids Neochonetes (N.) granulifer (Owen) and Neochonetes (N.) mixteco n. sp. have been found in the Ixtaltepec Formation (Lower-Middle Pennsylvanian), Oaxaca, southeastern Mexico.

The find of Yagonia in Osagean rocks of Tamaulipas State represents the oldest record of the genus and it indicates paleogeographic relations between Australia, South America and North America. The report of Tornquistia confirms the presence of the genus in North America. Pennsylvanian Neochonetes $(N$.) species from Oaxaca State are related to typical North American lineages, the new species is included in that of Neochonetes (N.) carboniferus.
\end{abstract}

Keywords: Brachiopods, chonetids, Mississippian, Pennsylvanian, Tamaulipas, Oaxaca, Mexico.

\section{RESUMEN}

Se describen cuatro especies de chonétidos procedentes del Carbonífero de México. El anóplido Yagonia collinsoni Carter se ha recolectado en capas de la Formación Vicente Guerrero, del Osageense (Misisípico) de Tamaulipas, en el noreste de México. De la Formación Ixtaltepec, Pensilvánico Inferior-Medio de Oaxaca, en el sureste de México, se describen el anóplido Tornquistia sp. y los rugosochonétidos Neochonetes (N.) granulifer (Owen) y Neochonetes (N.) mixteco $\mathrm{n}$. sp.

La presencia del género Yagonia en el Osageense de Tamaulipas representa el registro más antiguo del género y señala relaciones paleogeográficas entre Australia, Suramérica y Norteamérica. El hallazgo de Tornquistia establece con certeza su existencia en Norteamérica. Las especies de Neochonetes $(N$.) que se describen se relacionan con linajes típicamente norteamericanos; la nueva especie se incluye en el grupo de Neochonetes $(N$.) carboniferus.

Palabras clave: Braquiópodos, chonétidos, Misisípico, Pensilvánico, Tamaulipas, Oaxaca, México.

\section{INTRODUCCIÓN}

Los afloramientos misisípicos del Cañón de la Peregrina, en el estado de Tamaulipas, y los pensilvánicos del área de Santiago Ixtaltepec, en Oaxaca, sobresalen por ser las secuencias sedimentarias, de esas edades, más com- pletas y fosilíferas que se han descrito para México, pese a lo cual, el conocimiento que se tiene sobre sus faunas es escaso y fragmentario. Para la región del Cañón de la Peregrina, como trabajos previos, existe la mención de algunos fósiles que indican la presencia de rocas con edades del Silúrico al Pérmico (Girty, 1926; Carrillo-Bravo, 
1961; Stewart et al., 1993, 1999), la descripción de los braquiópodos silúricos de la Formación Cañón de Caballeros (Boucot et al., 1997), el estudio de los braquiópodos atíridos, espiriféridos y espiriferínidos de la Formación Vicente Guerrero (la misma de la que proceden los chonétidos misisípicos del presente trabajo) (Sour Tovar et al., en prensa); además, se han descrito los fusulínidos de las rocas pérmicas de la región (Muir, 1936). Por lo que se refiere a las faunas pensilvánicas de la región de Santiago Ixtaltepec, se han descrito formalmente los diversos tipos de bivalvos que en ella se encuentran (QuirozBarroso y Perrilliat, 1997, 1998) y se han presentado algunas listas preliminares de los invertebrados pensilvánicos presentes en esta localidad y que poseen afinidad con faunas contemporáneas de la región del Continente Medio de Norteamérica (Quiroz-Barroso y Sour-Tovar, 1996; Sour Tovar et al., 1996).

En las faunas mencionadas, al igual que en otras que se han encontrado en rocas del Paleozoico Superior de México, los braquiópodos representan el grupo más sobresaliente por su abundancia relativa, por ser uno de los más diversificados y por la información estratigráfica y paleoambiental que brindan. En particular, los braquiópodos del Suborden Chonetidina son un grupo relativamente común dentro de las faunas misisípicas y pensilvánicas de las localidades mencionadas y su estudio representa una aportación para el conocimiento de las faunas paleozoicas de México y para el desarrollo de las bases paleontológicas que permitan establecer relaciones estratigráficas, paleobiogeográficas, paleoambientales o de otra índole entre los afloramientos paleozoicos del territorio mexicano y otras regiones del mundo.

\section{MARCO GEOGRÁFICO}

Los ejemplares de Yagonia collinsoni se recolectaron en la sección tipo de la Formación Vicente Guerrero, de edad misisípica (Osageense), y que se ubica (Fig. 1) en el Cañón de la Peregrina, 20 kilómetros al noroeste de Ciudad Victoria, capital del Estado de Tamaulipas. El acceso a la localidad es a través del camino que recorre el Cañón de la Peregrina, que se inicia en el kilómetro 8 de la carretera federal Ciudad Victoria-Monterrey y que atraviesa el poblado de La Libertad. Los afloramientos misisípicos se encuentran aproximadamente a 11 kilómetros del poblado de La Libertad, en los alrededores del Rancho Vicente Guerrero. La base de esta sección se presenta en el flanco izquierdo del arroyo San Felipe (234' 455 lat. N $-99^{\circ} 15^{\prime} 948$ long. O) y la cima en la Cuchilla del Monte (2346’392 lat. N-99¹6’054 long. O).

Tornquistia sp., Neochonetes (N.) granulifer y Neochonetes $(N$.) mixteco $\mathrm{n}$. sp. se recolectaron en rocas de la sección tipo de la Formación Ixtaltepec, de edad pensilvánica (Morroviense-Desmoinesiense). Dicha sec-
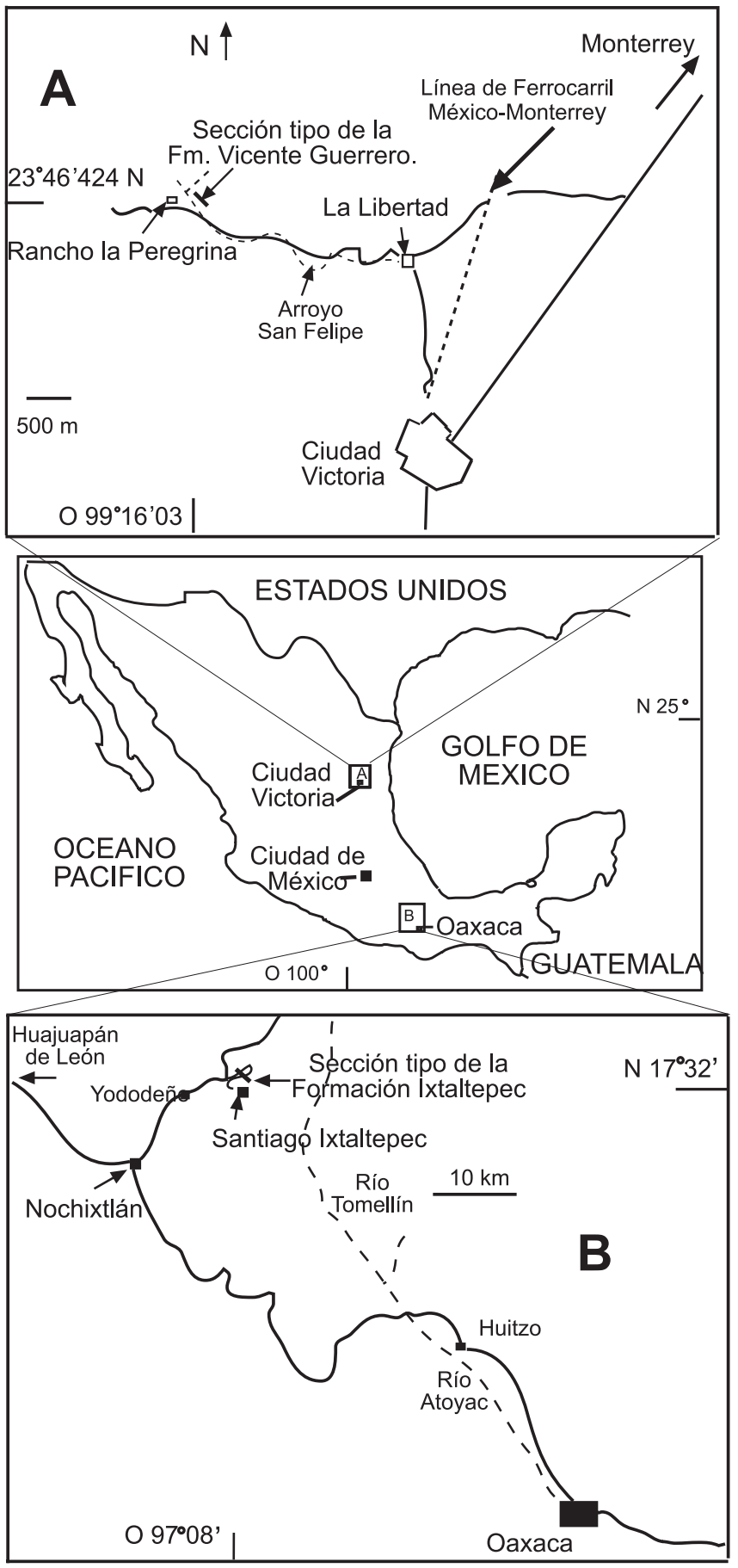

Figura 1. Localización de las zonas de procedencia del material descrito: A, ubicación de la sección tipo de la Formación Vicente Guerrero (Misisípico) en el área del Cañón de la Peregrina, norte de Ciudad Victoria, Tamaulipas; B, ubicación de la sección tipo de la Formación Ixtaltepec (Pensilvánico) en la región de Santiago Ixtaltepec, Oaxaca.

Locality map showing the provenance of the material described herein: A, type-section of the Vicente Guerrero Formation (Mississippian), Cañón de la Peregrina, N Ciudad Victoria, Tamaulipas; B, typesection of the Ixtaltepec Formation (Pennsylvanian), Santiago Ixtaltepec, Oaxaca. 
ción se encuentra (Fig. 1) en el Arroyo de las Pulgas, entre los $17^{\circ} 30^{\prime}$ y $17^{\circ} 35^{\prime}$ latitud Norte y entre los $97^{\circ} 05^{\prime}$ y $97010^{\prime}$ longitud Oeste, aproximadamente 500 metros al norte del pequeño poblado de Santiago Ixtaltepec, en el Municipio de Nochixtlán, Oaxaca. El acceso a la localidad es por medio del camino rural que se origina en el poblado de Nochixtlán y que accede a Santiago Ixtaltepec.

\section{MARCO ESTRATIGRÁFICO}

\section{ÁREA DEL CAÑÓN DE LA PEREGRINA, TAMAU- LIPAS}

En esta región, el basamento lo constituye el Gneis del Novillo, con rocas del Proterozoico Medio, del que se han obtenido edades radiométricas de 860 a 903 millones de años (Fries et al., 1962; Ruiz et al., 1988) y de hasta 1140 millones de años (Garrison, 1978). Descansando sobre la secuencia precámbrica, se encuentra el Esquisto Granjeno (Carrillo-Bravo, 1961; Cserna et al., 1977; Cserna y Ortega-Gutiérrez, 1978; Ramírez-Ramírez, 1992; OrtegaGutiérrez et al., 1993) que consiste de una mica-esquisto intercalada con anfibolita, pedernal y cuerpos de serpentinita. Las edades isotópicas que se han obtenido (Ramírez-Ramírez, 1992) poseen rangos de 257 a 330 millones de años y establecen un evento metamórfico para el Paleozoico Medio. La secuencia paleozoica de esta área posee un espesor de casi 1.700 metros de rocas de origen marino con edades que abarcan del Silúrico Superior al Pérmico. Las rocas silúricas, caliza, lutita y arenisca rica en cuarzo, se han agrupado en la Formación Cañón de Caballeros, con un espesor máximo de 100 metros (Gursky, 1996), y en ella se ha encontrado una rica fauna de invertebrados marinos con afinidad gondwánica (Boucot et al., 1997; Stewart et al., 1993, 1999).

Sobreyaciendo a las rocas silúricas, la Formación Vicente Guerrero (Fig. 2), en su sección tipo, presenta un espesor de cerca de 110 metros y está constituida, de la base al techo, por 40 metros de arenisca rojiza con dique-estratos de riolita en la parte superior, 40 metros de arenisca de color café con intercalaciones de lutita y 30 metros de una alternancia de arenisca, arenisca arcillosa, lutita, y calcarenita con bancos lenticulares formados por restos principalmente de braquiópodos. A lo largo de toda la secuencia misisípica, principalmente en los estratos de arenisca, se encuentran, en diversas concentraciones, invertebrados marinos con una clara dominancia de braquiópodos, en diversidad y abundancia, encontrándose entre ellos Lamellosathyris lamellosa (Léveillé, 1835) y Torynifer pseudolineatus (Hall, 1858) que indican la edad osageense para esta unidad.

Sobre la Formación Vicente Guerrero, en el Cañón de la Peregrina, se encuentran 1.100 metros de sedimentos marinos, de edad pérmica, de la Formación Guacamaya (Carrillo-Bravo, 1961) y sobre esta unidad descansan los

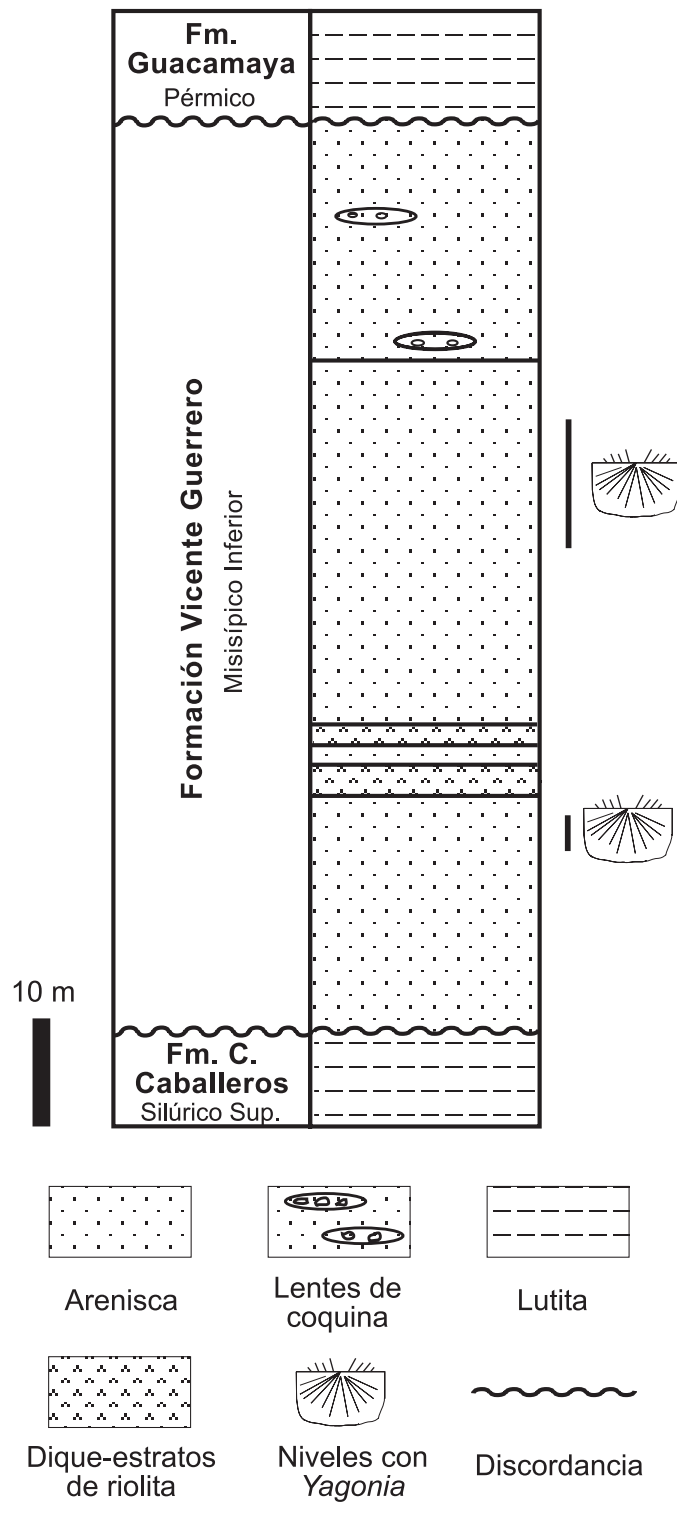

Figura 2. Sección tipo de la Formación Vicente Guerrero en el Cañón de la Peregrina y situación de las muestras con Yagonia collinsoni Carter, 1990.

Type-section of Vicente Guerrero Formation at the Cañón de la Peregrina showing the location of samples with Yagonia collinsoni Carter, 1990.

sedimentos jurásicos de origen continental del Grupo Huizachal (Rueda et al., 1993a, 1993b; Fastovsky et al., 1995; Reynoso y Clark, 1998; Stewart et al., 1999).

\section{ÁREA DE SANTIAGO IXTALTEPEC, OAXACA}

En está región, la secuencia paleozoica (Fig. 3) se encuentra sobre rocas metamórficas, principalmente esquistos, de edad precámbrica del Complejo Oaxaqueño. Sobre éste, discordantemente, se encuentra la Formación Tiñú, de edad tremadociense, dividida en dos miembros, el Inferior, con 13 metros de estratos de caliza de espesores variables, y el Superior, con 22 metros de lutita fina- 
mente laminada (Robison y Pantoja-Alor, 1968). En discordancia angular, la secuencia continúa con cerca de 67 metros de calcarenita y arenisca de la Formación Santiago, del Osageense (Misisípico) (Quiroz-Barroso et al., 2000; Navarro-Santillán et al., 2002).

Suprayaciendo a las rocas misisípicas, en un contacto discordante con rasgos de deformación tectónica, se encuentran cerca de 535 metros de rocas pensilvánicas (Navarro-Santillán et al., 2002). Los primeros 100 metros están constituidos por una lutita con intercalaciones de limolita, arenisca y caliza; los siguientes 105 metros son de arenisca, arenisca calcárea y calcarenita, y en los 330 metros de la parte superior dominan los estratos arcillosos con algunas intercalaciones de estratos delgados de arenisca o limolita. A todo lo largo de esta unidad se han recogido diversos fósiles pensilvánicos (MorrovienseDesmoinesiense) siendo los más representativos de la edad los braquiópodos Anthracospirifer occiduus (Sadlick, 1960) y Neospirifer dunbari King, 1933 (Sour-Tovar et al., 1996) y los bivalvos Permophorus spinulosa (Morningstar, 1922) y Myalinella meeki (Dunbar, 1924) (Quiroz-Barroso y Perrilliat 1997, 1998).

La secuencia paleozoica del área de Santiago Ixtaltepec concluye con un conglomerado de posible edad pérmica y que ha sido descrito como la Formación Yododeñe (Pantoja-Alor, 1970). Sobre ésta se encuentran rocas calcáreas del Cretácico, posiblemente Inferior.

\section{SISTEMÁTICA}

Los ejemplares que se describen se encuentran depositados en el Museo de Paleontología de la Facultad de Ciencias, Universidad Nacional Autónoma de México. Se numeran con el prefijo FCMP/M2 los recolectados en la sección tipo de la Formación Vicente Guerrero, Cañón de la Peregrina, Tamaulipas, y con el prefijo FCMP/E1, los recolectados en los afloramientos de la Formación Ixtaltepec, en el área de Santiago Ixtaltepec, Oaxaca. El estudio sistemático se hizo siguiendo la clasificación propuesta por Racheboeuf (2000) y los términos morfológicos son los utilizados por el mismo autor para chonétidos y por Williams y Brunton (1997).

FILO BRACHIOPODA Dumeril, 1806 SUBFILO RHYNCHONELLIFORMEA Williams et al., 1996

CLASE STROPHOMENATA Williams et al., 1996

ORDEN PRODUCTIDA Sarytcheva y Sokolskaya, 1959

SUBORDEN CHONETIDINA Muir-Wood, 1955

Superfamilia Chonetoidea Bronn, 1862

Familia Anopliidae Muir-Wood, 1962

Subfamilia Anopliinae Muir-Wood, 1962

Género Yagonia Roberts, 1976 (en Roberts et al.)

Especie tipo: Yagonia gibberensis Roberts, 1976 (en Roberts et al.).
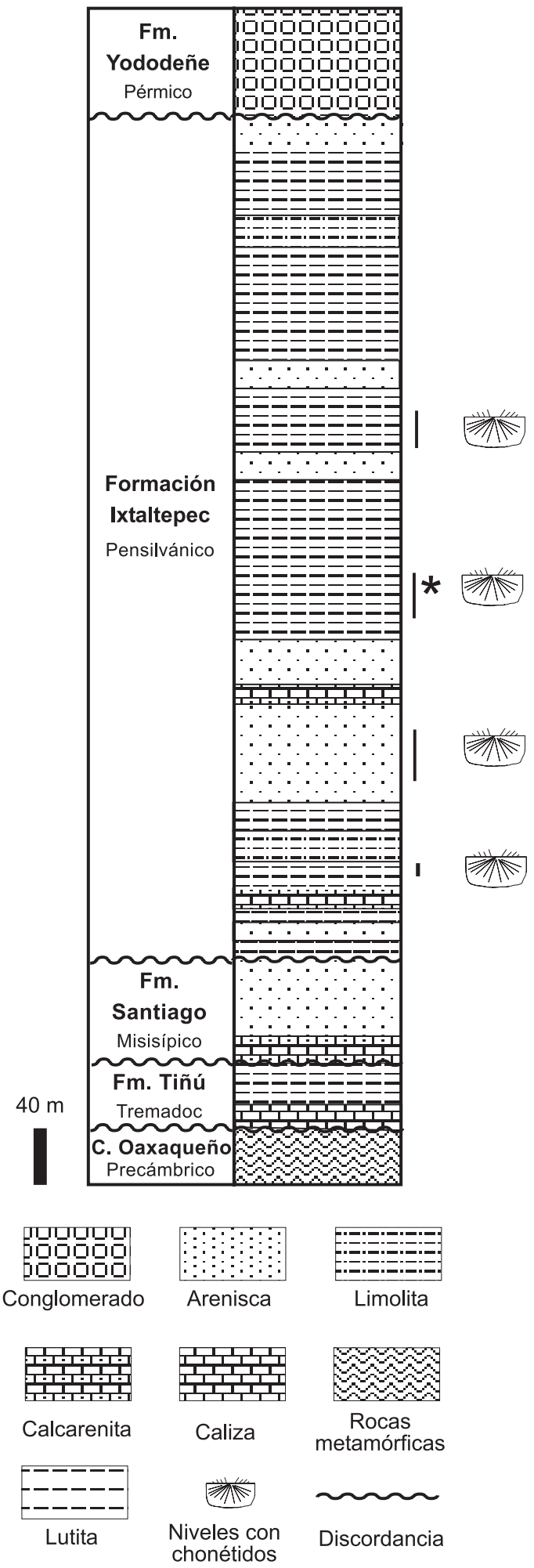

Figura 3. Columna estratigráfica de la sucesión paleozoica del área de Santiago Ixtaltepec, Oaxaca con la posición de los niveles en que se han recolectado los chonétidos. El asterisco indica el nivel de procedencia de los ejemplares de Neochonetes (N.) mixteco $\mathrm{n}$. sp. Stratigraphic section of the Paleozoic strata in Santiago Ixtaltepec area, Oaxaca, showing the location of chonetid-bearing beds. The beds with Neochonetes (N.) mixteco n. sp. are marked with an asterisk. 


\section{Yagonia collinsoni Carter, 1990}

Fig. 4a-h

*1990 Yagonia collinsoni n. sp.; Carter, 222, fig. 2.1-2.13.

Material: Cuatro moldes internos de valvas ventrales (FCMP/ M2-25a, b, c, FCMP/M2-29), una valva ventral parcialmente desconchada (FCMP/M2-26), tres moldes internos de valvas dorsales (FCMP/M2-28; FCMP/M2-30; FCMP/M2-299) y dos fragmentos de moldes externos de valvas dorsales (FCMP/M227; FCMP/M2-31). Estos ejemplares se recolectaron en la sección tipo de la Formación Vicente Guerrero en el Cañón de la Peregrina. Los estratos fosilíferos se encuentran a 18 y 47 metros de la base de la formación (Fig. 2).

Descripción: Concha de tamaño grande (el ejemplar mayor mide 13,5 mm de longitud por $18 \mathrm{~mm}$ de anchura), concavoconvexa, de contorno semiovalado o subrectangular redondeado, con la anchura mayor en el tercio posterior; las aurículas son pequeñas y aplanadas; no presenta pliegue ni seno medios. La superficie es lisa, sólo con líneas concéntricas de crecimiento; en los ejemplares parcialmente desconchados, se observan capilas radiales.

La valva ventral es moderadamente convexa, en mayor grado en la región cercana al umbo; el gancho es pequeño, apenas saliente por detrás de la interárea; la interárea es relativamente alta, apsaclina, en su interior presenta al menos 9 túbulos de espinas a cada lado; los más próximos al gancho están muy juntos y se dirigen hacia él; hacia los extremos están más espaciados y su dirección va cambiando gradualmente hasta llegar a ser posterior (Fig. 4a-c). En su interior presenta un septo medio muy largo y robusto que alcanza más de cuatro quintos de la longitud de la valva, llegando hasta la cresta marginal; el campo muscular es muy grande y está limitado por una cresta marginal baja pero neta; los aductores son pequeños, estrechos y alargados; los diductores son muy grandes, flabeliformes y presentan numerosas elevaciones radiales que alcanzan la cresta marginal (Figs. 4a-d, g); por delante de dicha cresta, los márgenes anterior y laterales llevan finas cóstulas radiales.

La valva dorsal es ligeramente cóncava, con las aurículas aplanadas. Interior con una cresta marginal baja, similar a la de la valva opuesta; proceso cardinal fuerte y largo, bilobulado, que se apoya en un septo medio, fuerte y corto, que se extiende sólo hasta el comienzo de los septos accesorios; sin alveolo; fosetas profundas, limitadas por crestas internas fuertes y altas; anderidios cortos; presenta un par de septos accesorios divergentes, fuertes, que se originan delante del septo medio y alcanzan la cresta marginal (Fig. 4e-f, h); lateralmente están flanqueados por fuertes tubérculos dispuestos en filas radiales, unidos en ocasiones formando septos adicionales más bajos que los centrales; márgenes con finas cóstulas, como los de la valva ventral.

Discusión: El género Chilenochonetes Isaacson y Dutro, 1999, del Turnesiense del $\mathrm{N}$ de Chile, es parecido a Yagonia por su forma y gran parte de los caracteres del interior dorsal. Se diferencia por su ornamentación capilada, por la ausencia en el interior de ambas valvas de una cresta marginal, por su interior ventral con septo medio mucho menos desarrollado y ausencia de elevacio-
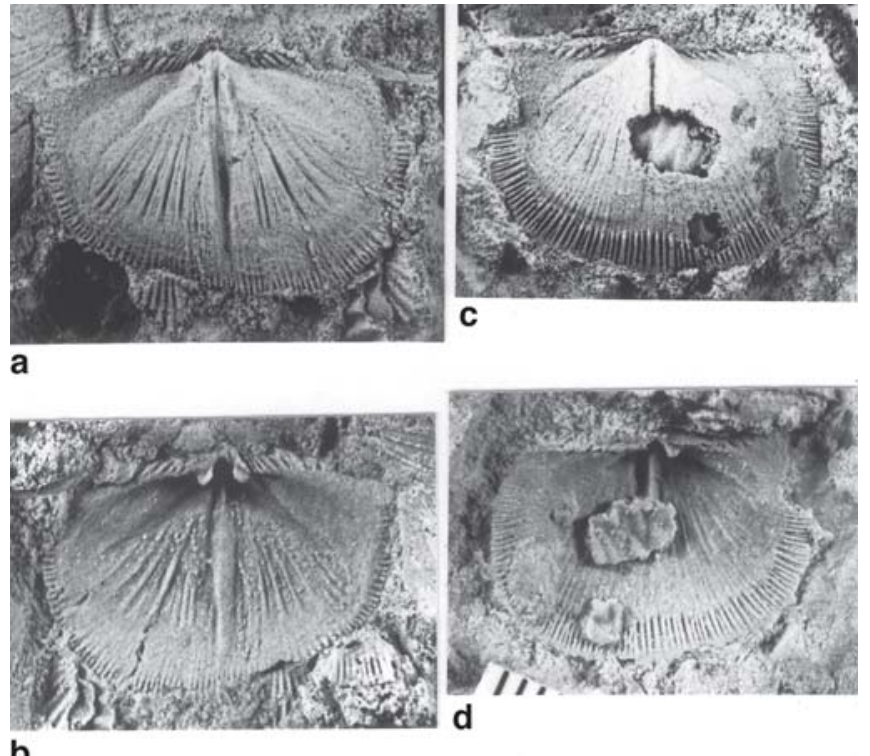

b
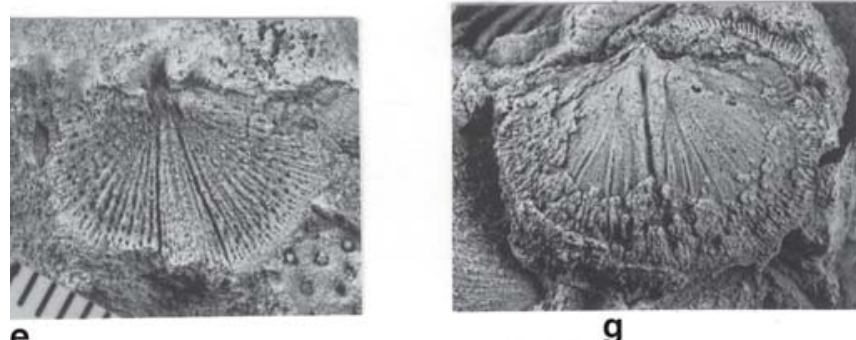

g

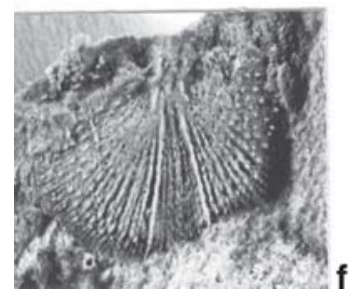

$1 \mathrm{~cm}$

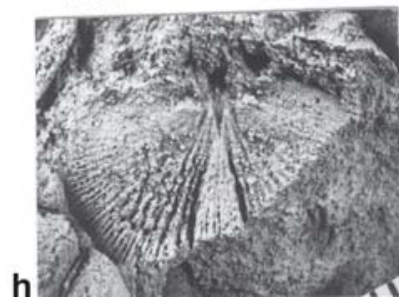

Figura 4. Yagonia collinsoni Carter, 1990. Formación Vicente Guerrero, Cañón de la Peregrina, Osageense inferiormedio. x2. a-b, Molde interno ventral y látex, FCMP/ M2-25a. c-d, Molde interno ventral y látex, FCMP/ M2-25b. e-f, Molde interno dorsal y látex, FCMP/ M2-28. g, Molde interno ventral FCMP/M2-29. h, Molde interno dorsal FCMP/M2-30.

Yagonia collinsoni Carter, 1990. Vicente Guerrero Formation, Cañón de la Peregrina, lower-middle Osagean. x2. $\boldsymbol{a}-\boldsymbol{b}$, Ventral internal mould and latex cast, FCMP/M2-25a. c-d, Ventral internal mould and latex cast, FCMP/M2-25b. e-f, Dorsal internal mould and latex cast, FCMP/M2-28. g, Ventral internal mould, FCMP/M2-29. h, Dorsal internal mould, FCMP/M2-30.

nes radiales en las huellas de los diductores, y por la presencia de un alveolo en el interior dorsal.

Yagonia collinsoni es muy similar a Y. gibberensis, especie tipo del género, que se encontró en el Viseense tardío o Namuriense temprano del Este de Australia 
(Roberts et al., 1976). Ambas especies se diferencian por la región umbonal ventral más extensa y de gran convexidad de la especie tipo (Carter, 1990). No existen otras especies descritas del género.

Distribución: El género se conoce del Carbonífero (Misisípico y Pensilvánico) del E de Australia, del Misisípico (Osageense tardío) de Illinois y del Pérmico de Argentina (Roberts et al., 1976; Amos, 1979).

La especie se ha descrito para el Osageense tardío de Illinois (Carter, 1990). En este trabajo al material analizado se le asigna una edad de Osageense temprano-medio por su asociación con Lamellosathyris lamellosa (Léveillé, 1835), Cleiothyridina tenuilineata (Rowley, 1900) y Torynifer pseudolineatus (Hall, 1858) (Sour-Tovar et al., en prensa).

\section{Género Tornquistia Paeckelmann, 1930}

Especie tipo: Leptaena (Chonetes) polita M'Coy, 1855.

\section{Tornquistia sp.}

Fig. 5a-h

Material: Dos moldes externos e internos de valvas ventrales (FCMP/E1-1722a, b; FCMP/E1-1722e, f), un molde externo e interno de una valva dorsal (FCMP/E1-1722c, d), un molde interno dorsal y dos moldes externos dorsales (FCMP/E1-1940a, b, c) y un molde externo ventral (FCMP/E1-1940d), además de otros ejemplares fragmentarios no numerados. Este material se recolectó en la Formación Ixtaltepec, en su sección tipo del Arroyo de las Pulgas, 500 metros al norte del poblado de Santiago Ixtaltepec, Oaxaca (Fig. 3).

Descripción: Concha pequeña, concavoconvexa, con las aurículas aplanadas, transversa (FCMP/E1-1940d, el ejemplar más grande, mide: $\mathrm{L}=4 \mathrm{~mm}$; $\mathrm{A}=6 \mathrm{~mm})$, de contorno redondeado rectangular o subtriangular; máxima anchura en la charnela, sin seno ni pliegue medio; superficie lisa, sin que se observen líneas de crecimiento debido a la conservación.

Valva ventral convexa, con la región media abombada, al menos lleva dos espinas, rectas, a cada lado del umbo. Interior con un septo medio corto y delgado.

Valva dorsal suavemente cóncava. Interior con proceso cardinal pequeño y alveolo relativamente profundo y alargado; un par de septos accesorios divergentes, relativamente fuertes, se inician por delante del alveolo y terminan sin alcanzar el margen anterior; sin septo medio; anderidios no observados; superficie interna, salvo las aurículas, cubierta de tubérculos dispuestos en filas radiales.

Discusión: El material se asigna a Tornquistia por las características de su interior dorsal, especialmente la presencia del par de septos accesorios, y por su forma y superficie lisa. Anopliopsis Girty, 1938, con una forma y ornamentación similar, se distingue por presentar un seno ventral y varios pares de septos laterales en ambas valvas.
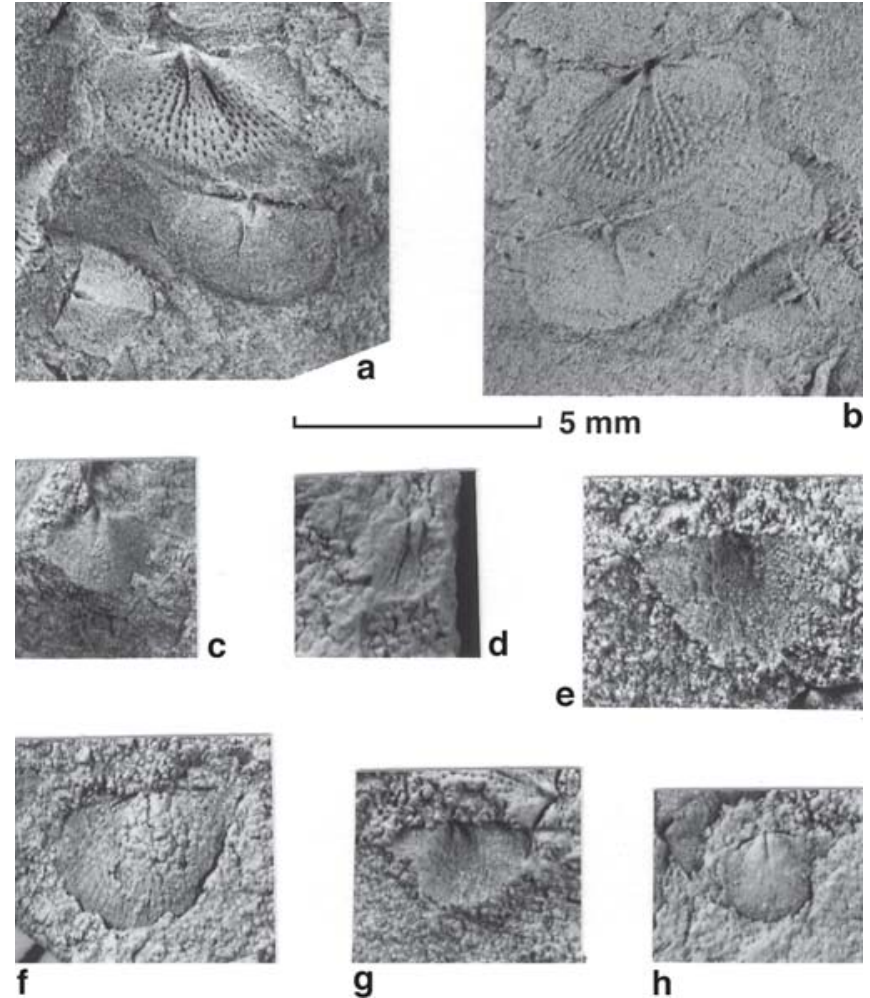

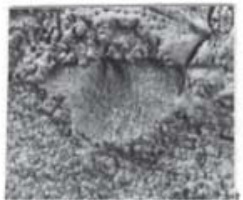

g h

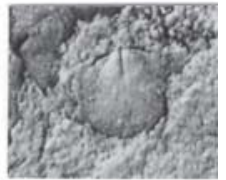

Figura 5. Tornquistia sp. Formación Ixtaltepec, N de Santiago Ixtaltepec, Oaxaca, Pensilvánico Inferior-Medio. x5. a-b, Molde interno dorsal, FCMP/E1-1940a (arriba) y dos moldes externos dorsales, FCMP/E11940b, c (abajo) (a), y látex de los mismos ejemplares (b). c-d, Moldes externo e interno de una valva dorsal, FCMP/E1-1722c, d. e-f, Moldes externo e interno de una valva ventral, FCMP/E1-1722e, f. gh, Moldes externo e interno de una valva ventral, FCMP/E1-1722a, b.

Tornquistia sp. Ixtaltepec Formation, N Santiago Ixtaltepec, Oaxaca, Lower-Middle Pennsylvanian. x5. $\boldsymbol{a}$-b, Dorsal internal mould, FCMP/E1-1940a (top), and two dorsal external moulds, FCMP/E1$1940 b, c$ (bottom) (a), and latex cast of the same specimens $(\boldsymbol{b}) . \boldsymbol{c}-\boldsymbol{d}$, External and internal moulds of a dorsal valve, FCMP/E1-1722c, d.e-f, External and internal moulds of a ventral valve, FCMP/E1-1722e, f. $\boldsymbol{g}$-h, External and internal moulds of a ventral valve, $F C M P / E 1-1722 a, b$.

La falta de ejemplares adecuados nos impide mayores precisiones.

El género Tornquistia tiene una amplia distribución estratigráfica (Devónico Superior-Pérmico). La distribución geográfica es también extensa, estando ampliamente distribuido en Europa, Asia y Australia; para América sólo se había citado en el Pérmico del Ártico de Canadá (Bamber y Waterhouse, 1971), el Carbonífero Superior de Argentina (Amos, 1960; Lech y Aceñolaza, 1987) y, con dudas, para el Misisípico de Texas (Carter, 1967). 
Distribución: A los ejemplares descritos de Tornquistia sp. se les asigna una edad de Pensilvánico Inferior-Medio (Morroviense-Desmoinesiense) por su asociación con Neochonetes (N.) granulifer, Anthracospirifer occiduus Sadlick, 1960 y Neospirifer dunbari King, 1933 (QuirozBarroso y Sour-Tovar, 1996; Sour-Tovar et al., 1996).

Familia Rugosochonetidae Muir-Wood, 1962 Subfamilia Rugosochonetinae Muir-Wood, 1962 Género Neochonetes Muir-Wood, 1962

Subgénero Neochonetes (Neochonetes) Muir-Wood, 1962

Especie tipo: Chonetes dominus King, 1938.

Discusión: Archbold (1981) describió dos grupos en el subgénero Neochonetes (Neochonetes), uno de ellos basado en $N$. (N.) granulifer, y que incluye entre otras a la especie tipo, y el otro basado en $N$. (N.) carboniferus (Keyserling, 1846). Martínez Chacón y Winkler Prins (2000) analizan las diferencias entre los dos grupos y encuentran que quizá la más importante sea el tipo de costulación, más débilmente desarrollada, llegando las cóstulas a ser inapreciables hacia el margen anterior, en el primer grupo.

En el Pensilvánico de la región de Santiago Ixtaltepec hemos reconocido la presencia de $N$. (N.) granulifer (obviamente, incluido en el grupo del mismo nombre) y de la nueva especie $N$. (N.) mixteco, que incluimos en el grupo de $N$. (N.) carboniferus.

\section{Neochonetes (Neochonetes) granulifer (Owen, 1852)}

Figs. 6, 7a-r, 8b, ñ-p

* 1852 Chonetes granulifera Owen, 583, lám. 5, figs. 12 a-d.

1932 Chonetes granulifer Owen; Dunbar y Condra, 138, lám. 18, figs. 1-10.

1932 Chonetes granulifer var. meekanus (Girty); Dunbar y Condra, 143, lám. 18, figs. 11-17.

1944 Chonetes granulifer Owen; Cooper, p. 347, lám. 134, figs. 24-26.

1962 Neochonetes granulifer Owen; Muir-Wood, 87, lám. 10, figs. 8 , 15; lám. 11, fig. 5 .

1968 Neochonetes granulifer (Owen); Sturgeon y Hoare, 37, lám. 9, figs. 1-19.

Material: Ejemplares figurados o medidos: Moldes externos e internos de dos valvas ventrales (FCMP/E1-1722g-h y FCMP/ E1-1722j-k), moldes externo e interno de una valva dorsal (FCMP/E1-17221-m), dos moldes externos dorsales con la interárea ventral (FCMP/E1-1721c, FCMP/E1-1944h), tres moldes externos ventrales (FCMP/E1-1721a, FCMP/E1-1722n, FCMP/E1-1941c), 12 moldes internos ventrales (FCMP/E11129, FCMP/E1-1722i, FCMP/E1-1940e-f, FCMP/E1-1941a-b, FCMP/E1-1944a-f), tres moldes externos dorsales (FCMP/E11940g-h, FCMP/E1-1944g), y un molde interno dorsal (FCMP/
E1-1721b), además de otros ejemplares incompletos, no numerados. Este material se recolectó en la Formación Ixtaltepec, en su sección tipo del Arroyo de las Pulgas, 500 metros al norte del poblado de Santiago Ixtaltepec, Oaxaca (Fig. 3).

Descripción: Concha de tamaño medio (el ejemplar mayor mide 12,4 $\mathrm{mm}$ de longitud y $19 \mathrm{~mm}$ de anchura; las dimensiones se pueden encontrar en la Fig. 6), concavoconvexa a planoconvexa, de contorno subrectangular, transversa (longitud/anchura aproximadamente 0,6); máxima anchura en la charnela o ligeramente por delante de ella; sin seno ni pliegue medio. Ornamentación multicostelada, con costillas y cóstulas finas, 5-6 por mm en el margen anterior, que se atenúan hacia el frente, cruzadas por ocasionales líneas concéntricas de crecimiento, más frecuentes en la región anterior.

Valva ventral moderadamente convexa, con la máxima curvatura en la región posterior, desde donde disminuye regularmente hasta el frente; aurículas aplanadas; gancho apenas saliente; a cada lado del umbo hay al menos 7 bases de espinas (Fig. 7d), formando las más alejadas un ángulo grande con la charnela. Interior con un septo medio, fuerte y alto en su región posterior, entre los aductores, que se continúa hacia delante como una elevación baja, hasta el final de los diductores. Las huellas de los aductores están claramente impresas, son pequeñas, alargadas, estrechas y de contorno ovalado; las huellas de los diductores son grandes, flabeliformes y están mal definidas; por delante de los aductores se origina un par de crestas vasculares paralelas al septo medio, generalmente fuertes, que se extienden hasta cerca del margen anterior; toda la superficie interna, excepto el campo muscular, está cubierta por tubérculos formando filas radiales; en los ejemplares mayores, se aprecia un reborde marginal.

Valva dorsal ligeramente cóncava, con excepción de las aurículas, que son aplanadas; contorno semielíptico. Proceso cardinal bilobado, separado del septo medio por un alveolo; el septo medio se extiende hasta unos dos tercios de la longitud de la valva; anderidios más finos que el septo medio, cortos, divergentes formando un ángulo de cerca de $70^{\circ}$ (Fig. 7l,n);

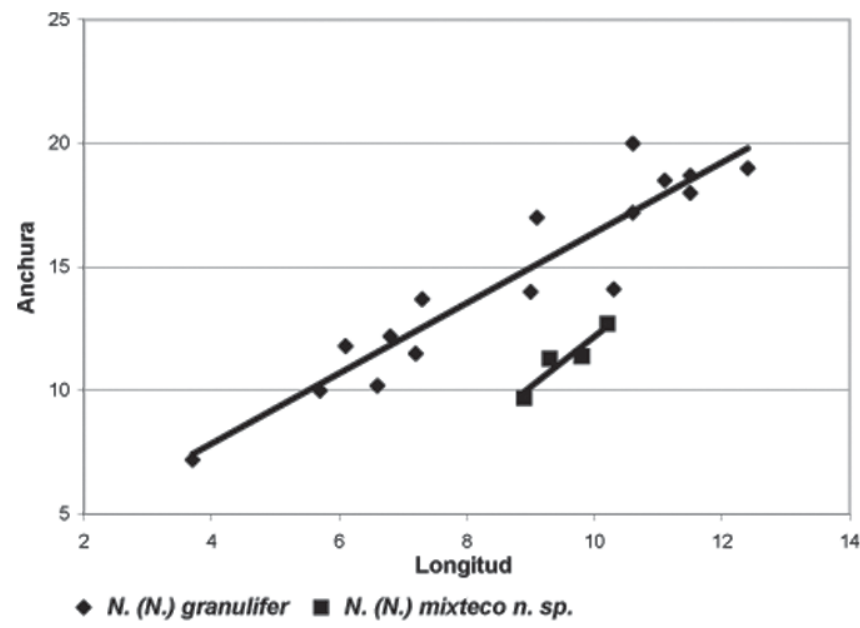

Figura 6. Diagrama longitud-anchura de Neochonetes (N.) granulifer (Owen, 1852) y N. (N.) mixteco n. sp. Length-width diagram of Neochonetes (N.) granulifer (Owen, 1852) and N. (N.) mixteco n. sp. 
fosetas dentales profundas, con crestas internas elevadas, formando en su inicio un ángulo muy pequeño con la charnela y continuándose una cierta distancia paralelas a la misma; crestas braquiales muy poco marcadas; aductores poco marcados, las huellas del par anterior, situadas entre el septo y el anderidio correspondiente, son pequeñas, alargadas, estrechas y de con- torno piriforme; las posteriores son inapreciables; superficie interna, excepto las aurículas, cubierta de tubérculos de manera similar a la valva ventral.

Discusión: La especie Neochonetes (N.) granulifer está ampliamente distribuida a lo largo del Pensilvánico de
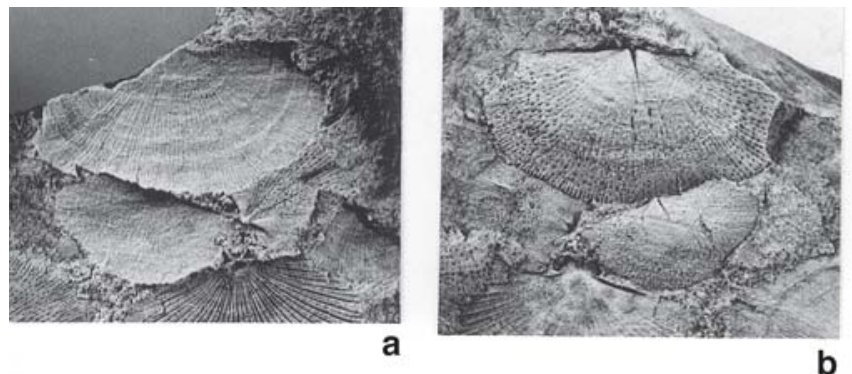

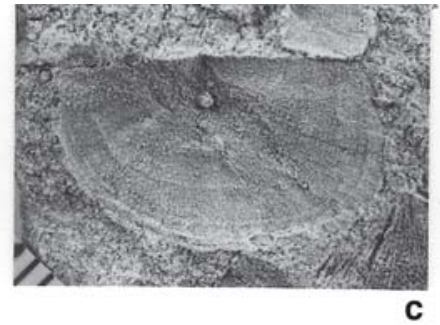

$1 \mathrm{~cm}$

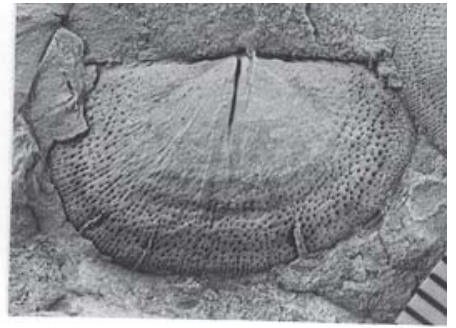

d
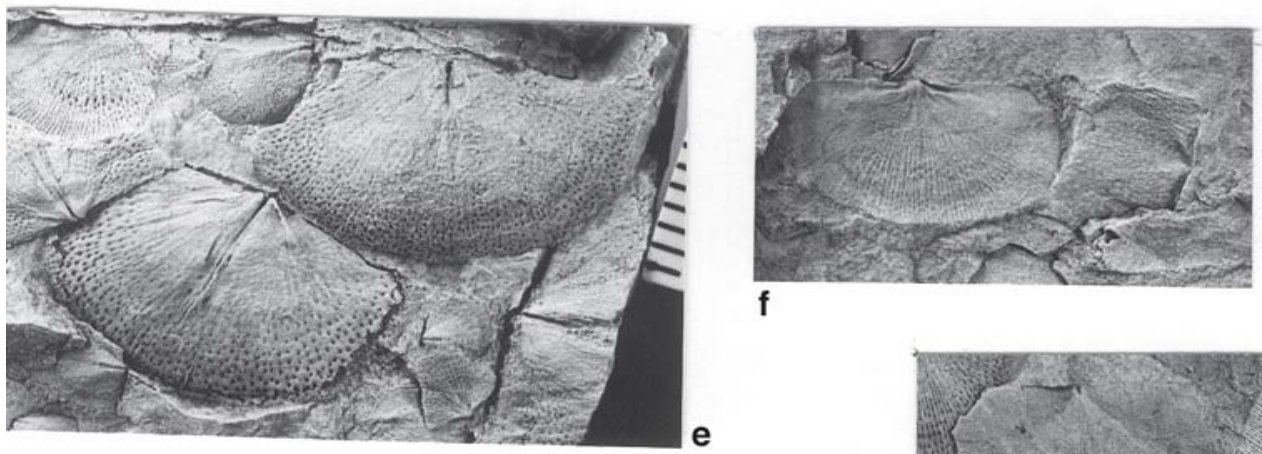

f

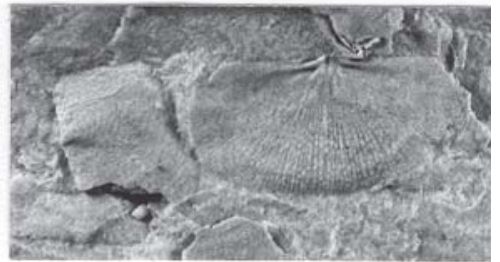

g
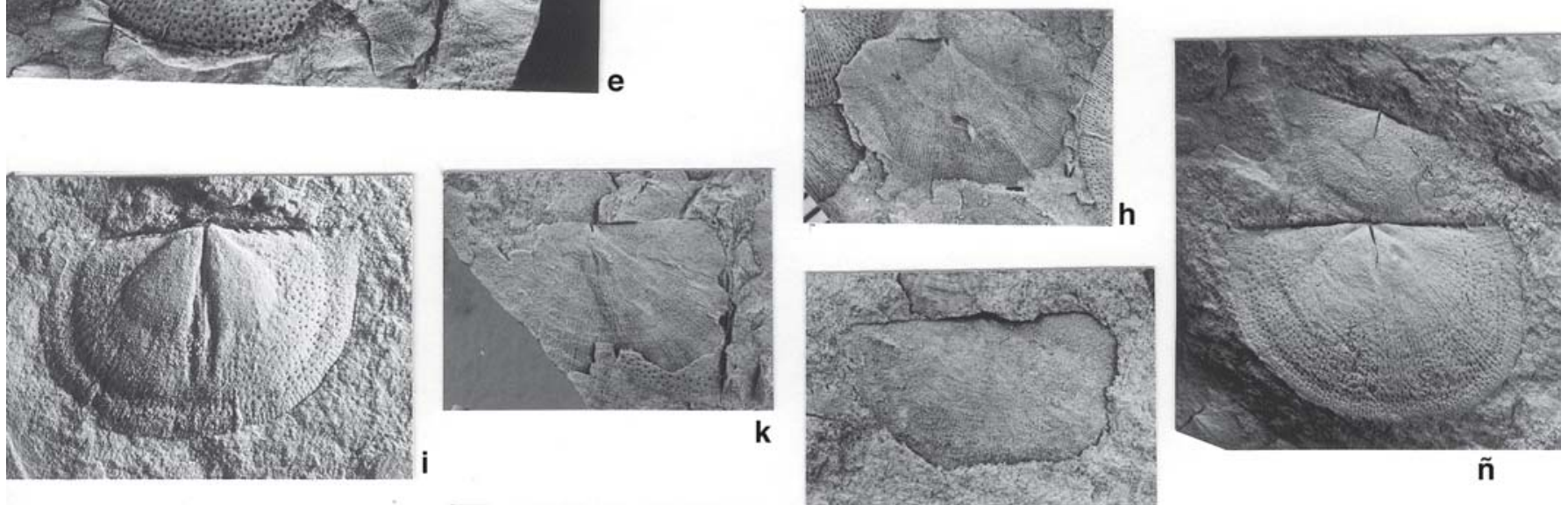

$\tilde{\mathbf{n}}$
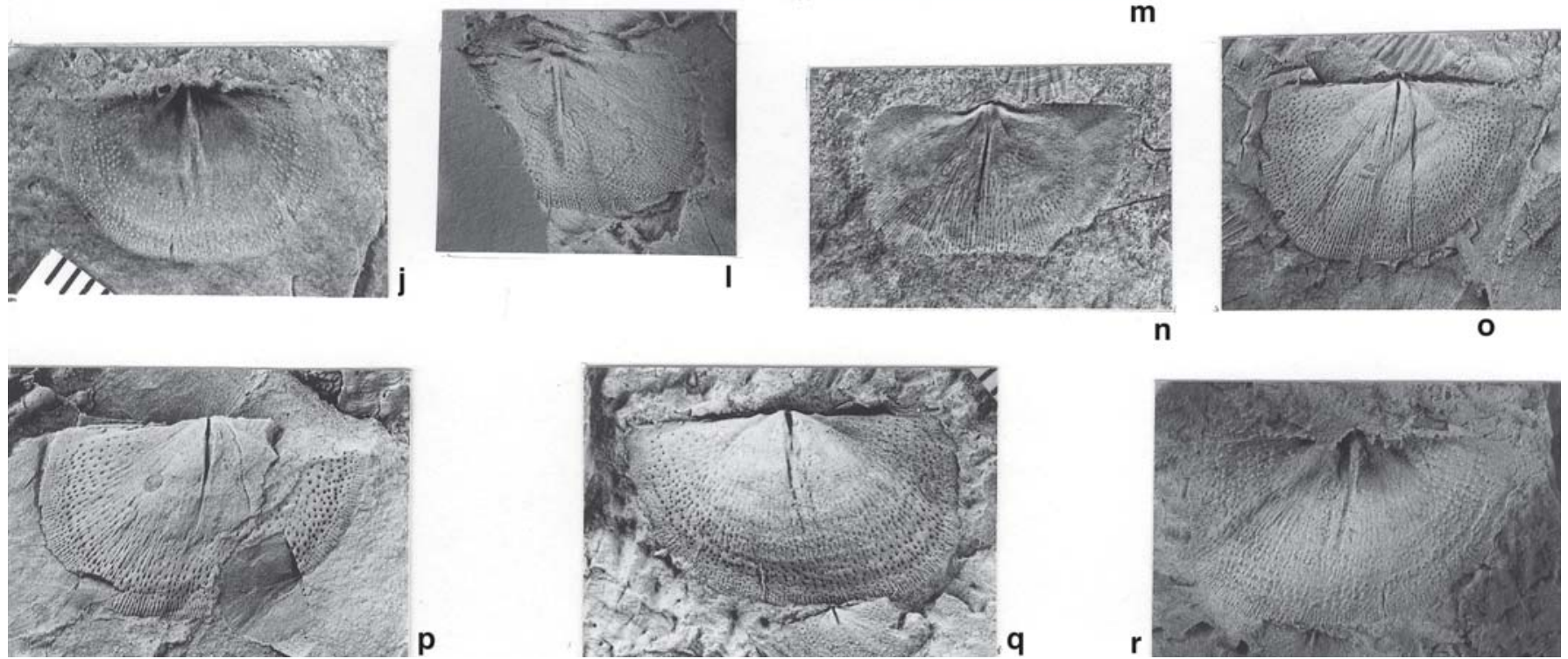
Norteamérica; su morfología es bastante variable, caracterizándose por su concha muy transversa, moderadamente concavoconvexa, con la máxima anchura en la charnela y ornamentación de cóstulas muy finas, que se atenúan hacia delante. Se parece a la especie tipo del género, de la que se diferencia por la menor curvatura de sus valvas. Neochonetes (N.) villamaninensis Martínez Chacón y Winkler Prins, 2000, del Moscoviense inferior de la Cordillera Cantábrica (España), tiene una forma y ornamentación similar, diferenciándose por la valva dorsal plana, menor ángulo entre los anderidios, características del septo medio dorsal y reborde marginal ventral más fuerte en la especie cantábrica. Las diferencias con la nueva especie $N$. (N.) mixteco se encuentran a continuación de la descripción de dicha especie.

Distribución: $N$. (N.) granulifer se ha recolectado en formaciones del Pensilvánico de Iowa, Nebraska, Missouri, Kansas (Archbold, 1981), Ohio (Sturgeon y Hoare, 1968), Indiana y Texas (Cooper, 1944), y en el Carbonífero Superior de Europa Occidental (Gran Bretaña y Alemania, Archbold, 1981). En la Formación Ixtaltepec se presenta a lo largo de toda la columna y se le ha asignado una edad de Pensilvánico Inferior-Medio (Morroviense-Desmoinesiense).

\section{Neochonetes (Neochonetes) mixteco $\mathrm{n} . \mathrm{sp}$.} Figs. 6, 7f-g, 8a-q

Etimología: De mixteco, habitante de la región mixteca, norte y noreste del Estado de Oaxaca (sureste de México).

Localidad tipo y estrato tipo: Arroyo de las Pulgas, 500 metros al norte del poblado de Santiago Ixtaltepec, Oaxaca. Estrato de lutita gris verdosa situado aproximadamente 245 metros sobre la base de la Formación Ixtaltepec.
Material: Holotipo, FCMP/E1-1940i, molde interno ventral (Fig. 8a, b), moldes interno y externo de una valva dorsal (FCMP/E1-1722o-p), más tres moldes externos ventrales (FCMP/E1-1721e-i), dos moldes internos ventrales (FCMP/E11944i-j), dos moldes externos dorsales (FCMP/E1-1721d, FCMP/E1-1941d) y tres moldes internos dorsales (FCMP/E11721f-g, FCMP/E1-1722q). Todos los ejemplares proceden de la Formación Ixtaltepec, en su sección tipo del Arroyo de las Pulgas, 500 metros al norte del poblado de Santiago Ixtaltepec, Oaxaca.

Diagnosis: Neochonetes (Neochonetes) del linaje de $N$. (N.) carboniferus, con la concha muy delgada, ligeramente transversa, de forma subcuadrada redondeada, máxima anchura un poco por delante de la charnela; valva ventral débilmente convexa, originando una cavidad del corpus muy somera; seno y pliegue medio débilmente desarrollados o ausentes; ornamentación de costillas y cóstulas muy finas, unas 8 por $\mathrm{mm}$ en el margen anterior, claramente desarrolladas sobre ambas valvas. Interior dorsal con anderidios formando un ángulo de unos $40^{\circ}$.

Neochonetes (Neochonetes) of the lineage of N. (N.) carboniferus with the shell very thin, slightly transverse, of rounded subquadrate outline, and with the gratest width slightly in front of the hinge line; ventral valve weakly convex, giving rise to a shallow corpus cavity; sulcus and median fold weakly developed or absent; ornamentation of fine but distinct costae and costellae, c. 8 per $\mathrm{mm}$ at the anterior margin. Dorsal interior with anderidia forming an angle of $c .40^{\circ}$.

Descripción: Concha de tamaño medio (dimensiones del holotipo: $\mathrm{L}=9,8 \mathrm{~mm}, \mathrm{~A}=11,4 \mathrm{~mm}$; las dimensiones de otros ejemplares se encuentran en la Fig. 6), débilmente concavoconvexa, ligeramente transversa (L/A alrededor de 0,85 ), de contorno subcuadrado redondeado, con las extremidades cardi-

Figura 7. a-e, Neochonetes (N.) granulifer (Owen, 1852). a-b, Moldes externos (a) e internos (b) de dos valvas ventrales, FCMP/ E1-1722g, h (ejemplar grande), FCMP/E1-1722j, k (ejemplar pequeño). c, Molde externo ventral, FCMP/E1-1941c. d, Molde interno ventral, FCMP/E1-1941e (látex en Fig. 8b). e, Moldes internos de dos valvas ventrales, FCMP/E1-1944b, c. f-g, Molde interno dorsal de Neochonetes (N.) granulifer (FCMP/E1-1721b) y molde externo ventral de Neochonetes (N.) mixteco n. sp. (FCMP/E1-1721i) (f), y látex de los mismos ejemplares (g). h-r, Neochonetes (N.) granulifer. h, Molde externo dorsal, FCMP/E1-1940h. i-j, Molde interno ventral y réplica en látex, FCMP/E1-1129. k-l, Molde externo dorsal, y látex del molde interno dorsal del mismo ejemplar, FCMP/E1-1940g. m-n, Moldes externo e interno de una valva dorsal, FCMP/E1-17221, m. ̃̃ , Moldes internos de dos valvas ventrales, FCMP/E1-1941a, b. o-p, Moldes internos ventrales de dos individuos, FCMP/E1-1940f, 1944d. q-r, Molde interno ventral y látex, FCMP/E1-1944a. Todos los ejemplares proceden de la Formación Ixtaltepec, N de Santiago Ixtaltepec, Oaxaca, Pensilvánico Inferior-Medio. x2.

$\boldsymbol{a}-\boldsymbol{e}$, Neochonetes (N.) granulifer (Owen, 1852). $\boldsymbol{a}$-b, External (a) and internal (b) moulds of two ventral valves, FCMP/ E1-1722g, h (large specimen), FCMP/E1-1722j, k (small specimen). c, Ventral external mould, FCMP/E1-1941c.d, Ventral internal mould, FCMP/E1-1941e (latex cast on Fig. 8b). e, Internal moulds of two ventral valves, FCMP/E1-1944 b, c.f-g, Dorsal internal mould of Neochonetes (N.) granulifer (FCMP/E1-1721b) and ventral external mould of Neochonetes (N.) mixteco $n$. sp. $(F C M P / E 1-1721 i)(\boldsymbol{f})$, and latex cast of the same specimens $(\boldsymbol{g})$. $\boldsymbol{h}$-r , Neochonetes (N.) granulifer. $\boldsymbol{h}$, Dorsal external mould, FCMP/E1-1940h. i-j, Ventral internal mould, and latex cast, FCMP/E1-1129. k-l, Dorsal external mould, and latex cast of the dorsal internal mould of the same specimen, FCMP/E1-1940g. m-n, External and internal moulds of a dorsal valve, FCMP/E1-1722l, m. $\tilde{\boldsymbol{n}}$, Internal moulds of two ventral valves, FCMP/E1-1941a, b. o-p, Two ventral internal moulds, FCMP/E1-1940f, 1944d. q-r, Ventral internal mould, and latex cast, FCMP/E1-1944a. All from the Ixtaltepec Formation, N Santiago Ixtaltepec, Oaxaca, Lower-Middle Pennsylvanian. x2. 
nales redondeadas y la máxima anchura situada un poco por delante de la charnela; aurículas aplanadas; seno y pliegue medios débilmente desarrollados o ausentes; ornamentación de costillas y cóstulas muy finas, unas 8 por milímetro en el margen anterior, claramente desarrolladas, cruzadas por líneas concéntricas de crecimiento, que en ocasiones son más fuertes. La concha es muy delgada, de tal forma que a veces, en los moldes, se mezclan las características externas (costillas y cóstulas) e internas (tubérculos) (Figs. 7f-g, 8e, h).

Valva ventral débil y uniformemente convexa, con el umbo muy poco saliente; a cada lado del umbo se sitúan al menos 7 bases de espinas, las más alejadas del umbo formando un án- gulo grande con la charnela. Interior con septo medio muy alto en su parte posterior entre los aductores, que se continúa hacia delante como una cresta baja hasta cerca de la mitad de la longitud de la valva; campo muscular débilmente impreso; los aductores son pequeños y ovalados y están rodeados por los diductores, más grandes y flabeliformes, reconocibles sólo por la ausencia de tubérculos; por delante de los aductores se sitúan un par de crestas vasculares paralelas al septo medio, que se continúan una cierta distancia por delante de él; presenta un reborde marginal bien desarrollado; la superficie interna, excepto el campo muscular, está cubierta de tubérculos dispuestos en filas radiales muy densamente empaquetados.

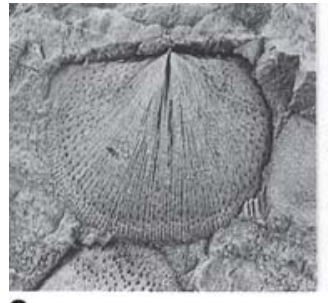

a

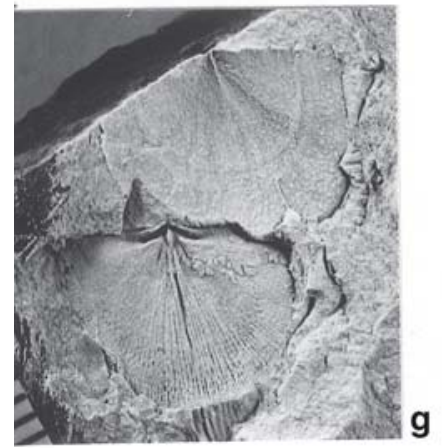

b

g
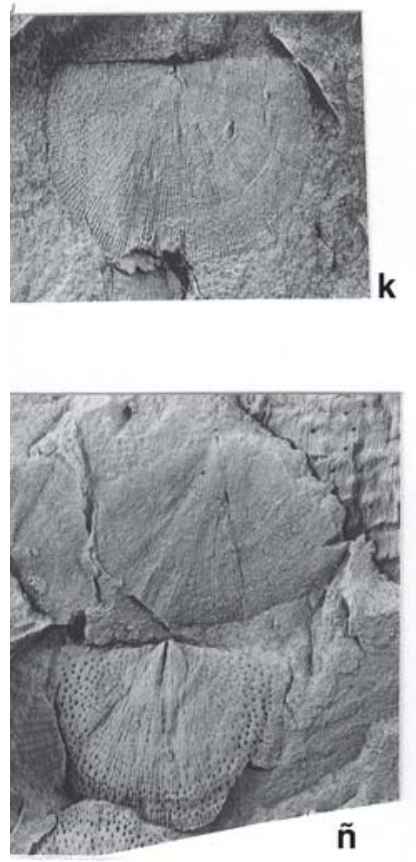
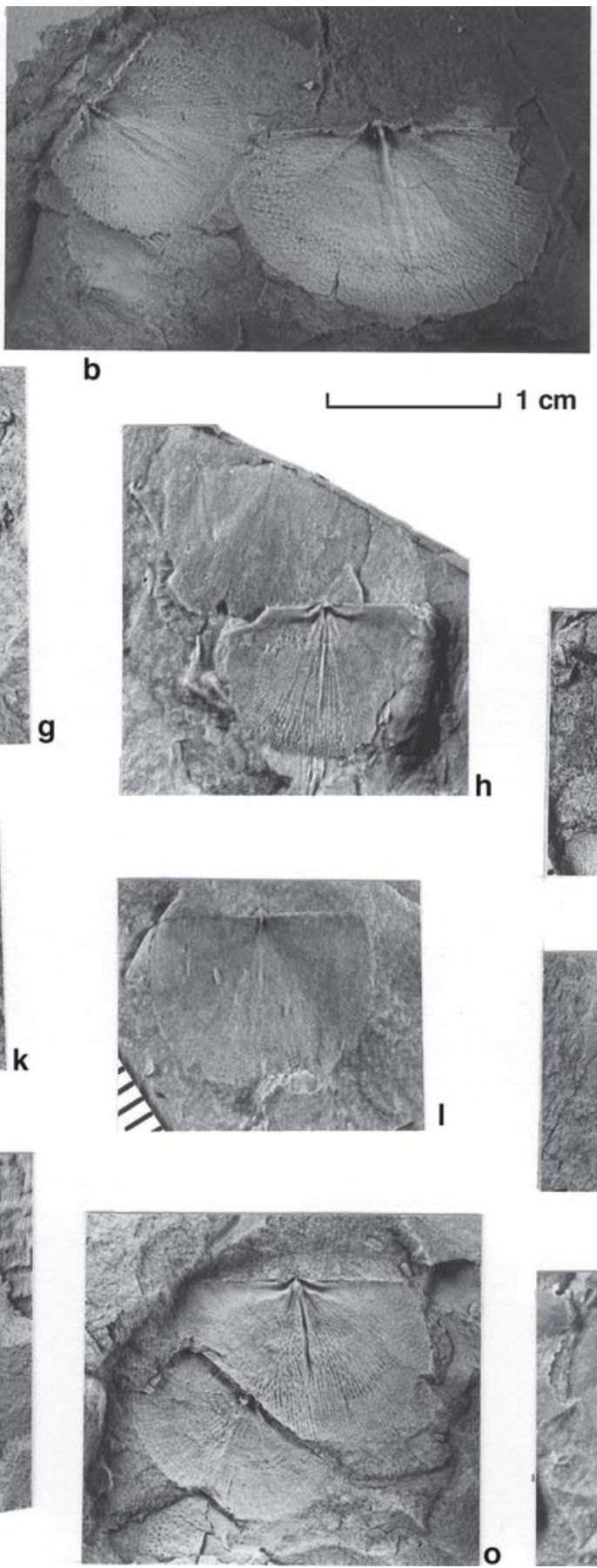
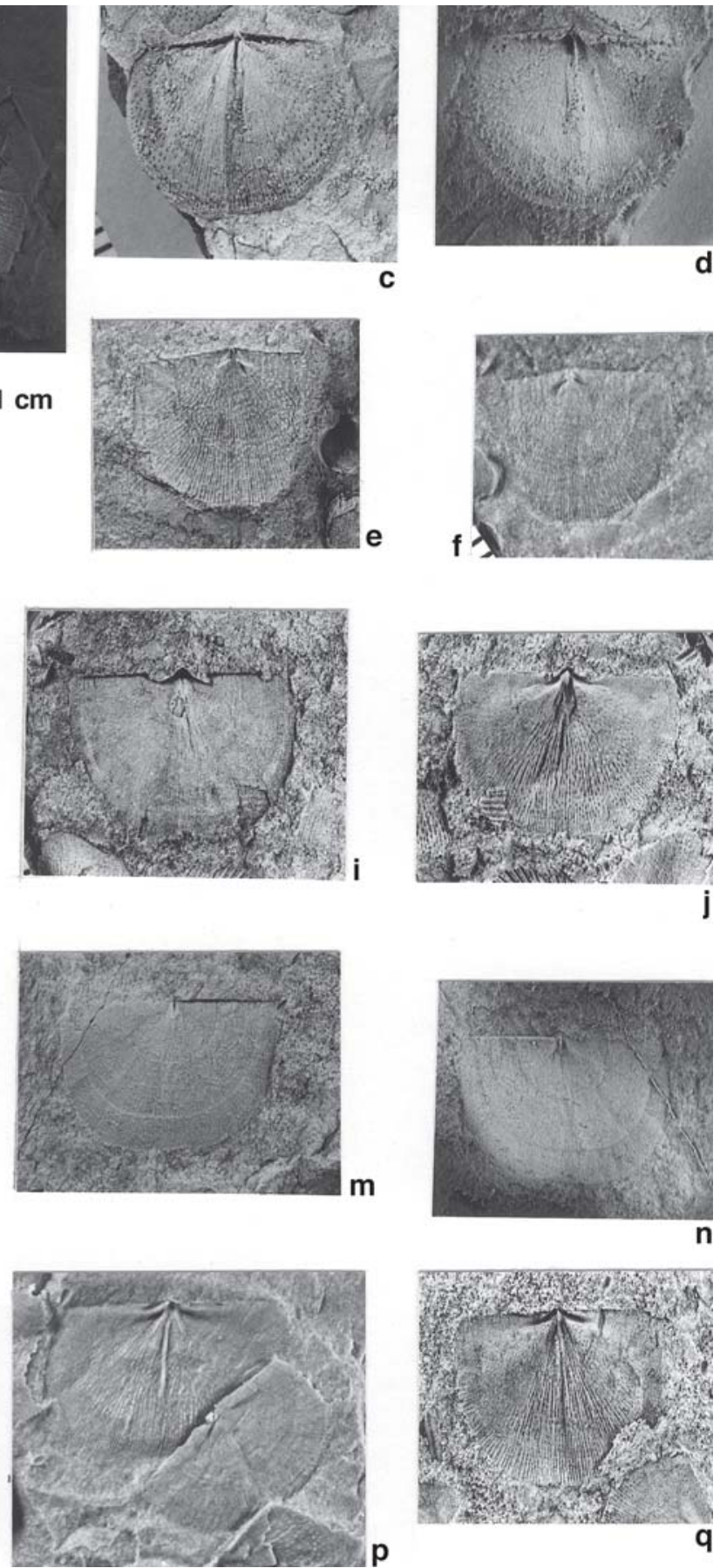
Valva dorsal débilmente cóncava, de contorno semielíptico. Interior con proceso cardinal separado por un alveolo largo y profundo de un septo medio, bajo y bastante largo, que alcanza más de la mitad de la longitud de la valva; anderidios delgados, divergentes según un ángulo de unos $40^{\circ}$ (Figs. 8g-h, o-q); fosetas dentales profundas, con crestas internas elevadas, que forman un ángulo de algo más de $20^{\circ}$ con la charnela y se extienden muy poco hacia los extremos; crestas braquiales poco patentes; aductores indistintos; superficie interna, excepto la región de las aurículas, cubierta de tubérculos similares a los de la valva ventral.

Discusión: Las características de la nueva especie, especialmente su interior ventral con desarrollo del par de crestas vasculares paralelas al septo medio, coinciden con las del género Neochonetes y, más concretamente, con las del subgénero $N$. (Neochonetes). Dentro de la subfamilia Rugosochonetinae, se asemeja al género Jakutochonetes Afanasjeva, 1977, del Carbonífero Superior del Ártico de Siberia, por su ornamentación muy fina y caracteres internos de ambas valvas, pero carece del surco medio en la parte anterior de las dos valvas, presente en el género siberiano. Por su delicada ornamentación, también recuerda al género Capillomesolobus Pecar, 1986 (subfamilia Capillomesolobinae, familia Rugosochonetidae), del Moscoviense-Pérmico de Norteamérica y de los Alpes Cárnicos, pero carece del seno bien desarrollado y provisto generalmente con una elevación media, característico de este último género, y ambos tienen interiores bastante diferentes.

$\mathrm{Su}$ concha casi equidimensional, con la anchura superando en muy poco a la longitud, y su valva ventral muy poco convexa, distinguen la nueva especie de $N$. (N.) carboniferus (Keyserling, 1846) y de $N$. (N.) babianus
Martínez Chacón y Winkler Prins, 2000 (del Bashkiriense y Moscoviense inferior de la Cordillera Cantábrica), a las cuales se asemeja porque sus cóstulas y costillas son netas hasta el frente de las valvas. Se diferencia de la especie norteamericana $N$. (N.) acanthophorus (Girty, 1934), además de por su concha muy poco transversa, por su pronunciada costulación, que no se borra hacia el margen anterior.

De $N$. (N.) granulifer, procedente de los mismos niveles, se distingue por su concha casi equidimensional, muy delgada, con ornamentación de cóstulas y costillas más finas pero que no se borran hacia delante, menor convexidad de la valva ventral, septo medio dorsal más bajo, anderidios formando un ángulo bastante menor, y crestas internas más divergentes con la charnela y continuándose poco hacia los lados.

Distribución: La especie se conoce sólo de la localidad tipo, capas de edad Pensilvánico Inferior-Medio (Morrowiense-Desmoinesiense).

\section{CONSIDERACIONES PALEOBIOGEOGRÁFICAS}

Yagonia collinsoni se ha encontrado en el Cañón de la Peregrina asociada a diversos géneros y especies de afinidad principalmente norteamericana. Su hallazgo representa el segundo registro de la especie para Norteamérica y el más antiguo del género que también se ha descrito para el Viseense tardío-Namuriense temprano del Este de Australia. En los afloramientos de este último continente,

Figura 8. a, Neochonetes (N.) mixteco n. sp. Holotipo, molde interno ventral, FCMP/E1-1940i. b, Réplica en látex del holotipo de N. (N.) mixteco (izquierda) y del molde interno ventral (derecha) del ejemplar de Neochonetes (N.) granulifer de la Fig. 7d (FCMP/E1-1941e). c-n, Neochonetes (N.) mixteco n. sp. c-d, Molde interno ventral y látex, FCMP/E1-1944i. e-f, Molde externo ventral y látex, FCMP/E1-1721e. g-h, Moldes externo ventral, FCMP/E1-1721h (arriba) e interno dorsal, FCMP/ E1-1721g (abajo) (g), y réplica en látex (h). i-j, Moldes externo e interno de una valva dorsal, FCMP/E1-1722p, o. k-l, Molde externo dorsal y látex, FCMP/E1-1721d. m-n, Molde externo dorsal y látex, FCMP/E1-1941d. ñ, Molde externo dorsal de Neochonetes (N.) granulifer (Owen, 1852), FCMP/E1-1944g (arriba), y molde interno ventral de Neochonetes (N.) mixteco n. sp., FCMP/E1-1944j (abajo). o-p, Molde interno dorsal de Neochonetes (N.) mixteco n. sp., FCMP/E1$1721 \mathrm{j}$ (arriba), y molde externo ventral de Neochonetes (N.) granulifer, FCMP/E1-1721a (abajo, izquierda) (o), y réplica en látex de los mismos ejemplares (p). q, Neochonetes (N.) mixteco n. sp. Molde interno dorsal, FCMP/E1-1722q. Todos los ejemplares proceden de la Formación Ixtaltepec, N de Santiago Ixtaltepec, Oaxaca, Pensilvánico Inferior-Medio. x2. $\boldsymbol{a}$, Neochonetes (N.) mixteco $n$. sp. Holotype, ventral internal mould, FCMP/E1-1940i. b, Latex cast of N. (N.) mixteco holotype (left) and the ventral internal mould of the specimen of Neochonetes (N.) granulifer FCMP/E1-1941e, figured on Fig. 7d. c-n, Neochonetes (N.) mixteco n. sp. $\boldsymbol{c}$-d, Ventral internal mould, and latex cast, FCMP/E1-1944i. e-f, Ventral external mould, and latex cast, FCMP/E1-1721e.g-h, Ventral external mould, FCMP/E1-1721h (top), and dorsal internal mould, FCMP/E1-1721g (bottom) (g), and latex cast $(\boldsymbol{h}) . i$-j, External and internal moulds of a dorsal valve, FCMP/E11722p, o. $\boldsymbol{k}$-l, Dorsal extrenal moul, and latex cast, FCMP/E1-1721d. m-n, Dorsal extrenal moul, and latex cast, FCMP/ E1-1941d. $\tilde{\boldsymbol{n}}$, Dorsal external mould of Neochonetes (N.) granulifer (Owen, 1852), FCMP/E1-1944g (top), and ventral internal mould of Neochonetes (N.) mixteco n. sp. FCMP/E1-1944j (bottom). o-p, Dorsal internal mould of Neochonetes (N.) mixteco $n$. sp. FCMP/E1-1721j (top), and ventral external mould of Neochonetes (N.) granulifer, FCMP/E1-1721a (left bottom) (o), and latex cast of the same specimens (p). q, Neochonetes (N.) mixteco $n$. sp. Dorsal internal mould, FCMP/E1-1722q. All from the Ixtaltepec Formation, N Santiago Ixtaltepec, Oaxaca, Lower-Middle Pennsylvanian. x2. 
al igual que en la Formación Vicente Guerrero, el género Yagonia se encuentra asociado al espiriférido Alispirifer. Para el género Alispirifer, Ivanova (1981) propuso un origen en Australia, una posterior dispersión por una vía hacia Suramérica y por otra hacia el este de Asia y Rusia, regiones en donde también se ha encontrado. La mayor antigüedad de los fósiles de Tamaulipas se contrapone a esta idea e indica un posible origen norteamericano para Yagonia y Alispirifer.

Tornquistia es un género con una amplia distribución geográfica y su presencia en México confirma su existencia en Norteamérica durante el Pensilvánico.

Neochonetes $(N$.$) granulifer y N$. (N.) mixteco n. sp. se asocian con formas típicas de la región del Continente Medio de Norteamérica y su presencia en la Formación Ixtaltepec es coherente con las relaciones paleobiogeográficas que se ha señalado que existen entre las faunas pensilvánicas de Oaxaca y del Continente Medio (QuirozBarroso y Sour-Tovar, 1996; Sour-Tovar et al., 1996; Quiroz-Barroso y Perilliat, 1997, 1998).

\section{AGRADECIMIENTOS}

Al Dr. Patrick Racheboeuf (Universidad de Brest) y a un revisor anónimo, por sus comentarios críticos al manuscrito. A Itzia E. Nieto López, Daniel Navarro Santillán y Leonora Martín Medrano, de la Facultad de Ciencias, Universidad Autónoma de México, por su asistencia técnica, y a Joaquín Vázquez Varela, del Departamento de Geología, Universidad de Oviedo, por la realización de las fotografías.

El primer autor (F. S. T.) agradece a la Dirección General de Asuntos del Personal Académico de la Universidad Nacional Autónoma de México por el apoyo otorgado para realizar la estancia de investigación en la Universidad de Oviedo, España, durante la cual se elaboró este trabajo, y por el soporte económico a través del proyecto PAPIIT IN228803; la segunda firmante (M. L. M. Ch.) agradece la ayuda económica de los proyectos MA-03-515-2, de la Universidad de Oviedo y BTE200301012, del Ministerio Español de Ciencia y Tecnología.

\section{REFERENCIAS}

Afanasjeva, G.A. 1977. Podotryad Chonetidina. In: Pozdnepaleozoyskie produktidy Sibiri i Arktiki (Ed. T.G. Sarytcheva). Akademiya Nauk SSSR, Trudy Paleontologicheskogo Instituta, 161, 5-41. (En ruso.)

Amos, A.J. 1960. Algunos Chonetacea y Productacea del Carbonífero Inferior y Superior del Sistema de Tepuel, provincia de Chubut. Revista de la Asociación Geológica Argentina, 15, 81-107.

Amos, A.J. 1979. Guía Paleontológica argentina. Parte I: Paleozoico. Sección V - Faunas Carbónicas. Sección VI: Faunas Pérmicas. Consejo Nacional de Investigaciones Científicas y Técnicas. Buenos Aires, 154 pp.

Archbold, N.W. 1981. Studies on Western Australian Permian brachiopods. 2. The Family Rugosochonetidae MuirWood, 1962. Proceedings Royal Society of Victoria, 93, 109-128.

Bamber, E.W. and Waterhouse, J.B. 1971. Carboniferous and Permian stratigraphy and paleontology, northern Yukon Territory, Canada. Bulletin of Canadian Petroleum Geology, 19, 29-250.

Boucot, A.J., Blodgett, R.B. and Stewart, J.H. 1997. European Province Late Silurian brachiopods from the Ciudad Victoria area, Tamaulipas, northeastern Mexico. In: Paleozoic sequence stratigraphy, biostratigraphy, and bigeography: Studies in honor of J. Granville ("Jess") Johnson (Eds. G. Klapper, M. A. Murphy and J. A. Talent). Geological Society of America, Special Paper 321, 273-293.

Carrillo-Bravo, J. 1961. Geología del anticlinorio Huizachal-Peregrina al NW de Ciudad Victoria, Tamaulipas. Boletín de la Asociación Mexicana de Geólogos Petroleros, 13, 1-98.

Carter, J.L. 1967. Mississippian brachiopods from the Chappel Limestone of Central Texas. Bulletins of American Paleontology, 53 (238), 488 pp.

Carter, J.L. 1990. New Brachiopods (Brachiopoda: Articulata) from the Late Osagean of the Upper Mississippi Valley. Annals of Carnegie Museum, 59, 219-247.

Cooper, G.A. 1944. Phylum Brachiopoda. In: Shimer, H.W. and Shrock, R. R. Index Fossils of North America. The Technological Press, Massachusets Institute of Technology. John Wiley and Sons Inc, New York, 277-365.

Cserna, Z. de and Ortega-Gutiérrez, F. 1978. Reinterpretation of isotopic age data from the Granjeno Schist, Ciudad Victoria, Tamaulipas y Reinterpretación tectónica del Esquisto Granjeno de Ciudad Victoria, Tamaulipas (Respuesta). Revista del Instituto de Geología, Universidad Nacional Autónoma de México, 2, 212-215.

Cserna, Z. de, Graf, J. L., Jr. y Ortega-Gutiérrez, F. 1977. Alóctono del Paleozoico Inferior en la región de Ciudad Victoria, Estado de Tamaulipas. Revista del Instituto de Geología, Universidad Nacional Autónoma de México, 1, 33-43.

Dunbar, C. O. and Condra, G. E. 1932. Brachiopoda of the Pennsylvanian System in Nebraska. Nebraska Geological Survey, Bulletin 5, 2 ser., 375 pp.

Fastovsky, D. E., Clark, J. M., Strater, N. H., Montellano, B. M., Hernández, R. R. and Hopson, J. A. 1995. Depositional environments of a Middle Jurassic terrestrial vertebrate assemblage, Huizachal Canyon, Mexico. Journal of Vertebrate Paleontology, 15, 561-575.

Fries, C., Jr., Schmitter, E., Damon, P. E., Livingston, D. E. y Erickson, R. 1962. Edad de las rocas metamórficas en los Cañones de la Peregrina y de Caballeros, parte centro-occidental de Tamaulipas. Boletín del Instituto de Geología, Universidad Nacional Autónoma de México, 64, 55-69.

Garrison, J. R., Jr. 1978. Reinterpretation of isotopic age data from the Granjeno Schist, Ciudad Victoria, Tamaulipas. Revista del Instituto de Geología, Universidad Nacional Autónoma de México, 2, 87-89.

Girty, G. H. 1926. A new area of Carboniferous rocks in Mexico. Science, 43(1.628), 286-287. 
Girty, G. H. 1934. New Carboniferous invertebrates. Journal Washington Academy of Sciences, 24, 251.

Girty, G.H. 1938. Descriptions of a new genus and a new species of Carboniferous brachiopods. Journal Washington Academia of Sciences, 28, 278-284.

Gursky, H.-J. 1996. Paleozoic stratigraphy of the Peregrina Canyon area, Sierra Madre Oriental, NE Mexico. Zentralblatt für Geologie und Paläontologie, Teil I, 1996 (5/ 6), 821-838.

Isaacson, P.E. and Dutro, T., Jr. 1999. Lower Carboniferous brachiopods from Sierra de Almeida, northern Chile. Journal of Paleontology, 73, 625-633.

Ivanova, E. A. 1981. Morfologiya i razvitie brakhiopod (Nadsemeistvo Paeckelmanellacea). Akademiya Nauk SSSR, Trudy Paleontologicheskogo Instituta, 195, 1-54. (En ruso.)

Keyserling, A. 1846. Wissenschaftliche Beobachtungen auf einer Reise in das Petschora-Land im Jhare 1843. Geognostische Beobachtungen. I. Palaeontologische Bemerkungen. St. Petersburg, 151-336.

King, R. H. 1938. New Chonetidae and Productidae from Pennsylvanian and Permian strata of North-central Texas. Journal of Paleontology, 12, 257-279.

Lech, R.R. y Aceñolaza, F.G. 1987. Braquiópodos en el Peñoniano (Carbónico Superior) de la Provincia de La Rioja. IV Congreso Latinoamericano de Paleontología, Bolivia, 1, 255-266.

Martínez Chacón, M.L. and Winkler Prins, C.F. 2000. New Rugosochonetidae (Brachiopoda) from the upper Bashkirian and Moscovian of the Cantabrian Mountains (N Spain). Revista Española de Paleontología, 15, 219-232.

M'Coy, F. 1855. Systematic descriptions of the British Palaeozoic fossils in the Geological Museum of the University of Cambridge, vol. 3. London, 407-661.

Muir, J. M. 1936. Geology of Tampico Region, Mexico. Thomas Murby and Company, London, England; American Association of Petroleum Geologist, Tulsa, Oklahoma, $280 \mathrm{pp}$.

Muir-Wood, H. M. 1962. On the morphology and classification of the brachiopod Suborder Chonetoidea. British Museum (Natural History), London, 132 pp.

Navarro-Santillán, D., Sour-Tovar, F. and Centeno-García, E. 2002. Lower Mississippian (Osagean) brachiopods from the Santiago Formation, Oaxaca, Mexico: stratigraphic and tectonic implications. Journal of South America Earth Sciences, 15, 327-336.

Ortega-Gutiérrez, F., López, R., Centeno-García, E. MoranZenteno, D. and Gómez-Caballero, A. (Eds.). 1993. PreMesozoic basement of NE Mexico, lower crust and mantle xenoliths of central Mexico and northern Guerrero terrane. First Circum-Pacific and Circum-Atlantic Terrane Conference. Instituto de Geología, Universidad Nacional Autónoma de México, Guidebook of Field Trip A, 96 pp.

Owen, D. D. 1852. Report of a Geological Survey of Wisconsin, Iowa and Minnesota and incidentally of a portion of Nebraska Territory. Lippincott, Grambo \& Company. Philadelphia, 638 pp.
Pantoja-Alor, J., 1970. Rocas sedimentarias paleozoicas de la región centro-septentrional de Oaxaca. In: Excursión México-Oaxaca. Sociedad Geológica Mexicana, Libro Guía (Eds. L. R. Segura y T. Rodríguez-Torres). México, 67-84.

Paeckelmann, W. 1930. Die Brachiopoden, des deutschen Unterkarbons. 1. Teil: Die Orthiden, Strophomeniden und Choneten des Mittleren und Oberen Unterkarbons. In: Die Fauna des deutschen Unterkarbons. 1. Teil. Abhandlungen der Preussischen Geologischen Landesantalt, N.F., 122, 143-326.

Pecar, J. 1986. Upper Carboniferous and Permian mesolobid chonetacean brachiopods of Karavanke Mountains (Yugoslavia) and Carnian Alps (Italy). Geologija, Ljubljana, 28/29, 9-53.

Quiroz-Barroso, S. A. and Perilliat, M. C. 1997. Pennsylvanian nuculoids (Bivalvia) from the Ixtaltepec Formation, Oaxaca, Mexico, Journal of Paleontology, 71, 400-407.

Quiroz-Barroso, S. A. and Perilliat, M. C. 1998. Pennsylvanian Bivalves from the Ixtaltepec Formation, Mexico, Journal of Paleontology, 72, 1011-1024.

Quiroz-Barroso, S. A. and Sour-Tovar, F. 1996. Paleogeographical implications of Pennsylvanian brachiopods and bivalves from Ixtaltepec Formation, Oaxaca, southeast Mexico. VI North American Paleontological Convention Abstracts Book. Washington D.C., U.S.A. Smithsonian Institution-Paleontological Society, 316.

Quiroz-Barroso, S. A., Pojeta, J., Jr., Sour-Tovar, F. and Morales-Soto, S. 2000. Pseudomulceodens: a Mississippian Rostroconch from Mexico. Journal of Paleontology, 74, 1184-1186.

Racheboeuf, P. R. 2000. Suborden Chonetidina. In: Treatise on Invertebrate Paleontology. Part H. Brachiopoda (Revised) 2 (Ed. R. Kaesler). New York and Lawrence, Geological Society of America and University of Kansas Press, 362-423.

Ramírez-Ramírez, C. 1992. Premesozoic geology of Huizachal-Peregrina Anticlinorium, Ciudad Victoria, Tamaulipas and adjacent parts of Eastern Mexico. Doctor of Philosophy Dissertation. The University of Texas at Austin, 317 pp. (inédito).

Reynoso, R. V. H. and Clark, J. M. 1998. A dwarf sphenodontian from the Jurassic La Boca Formation of Tamaulipas, México. Journal of Vertebrate Paleontology, 18, 197-225.

Roberts, J., Hunt, J. W. and Thompson, D. M. 1976. Late Carboniferous marine invertebrate zones of eastern Australia. Alcheringa, 1, 197-225.

Robison, R. and Pantoja-Alor, J. 1968. Tremadocian trilobites from the Nochixtlán region, Oaxaca, Mexico. Journal of Paleontology, 42, 767-800.

Rueda, G. J., López, O. E., Dueñas, M. A., y Rodríguez, J. L. 1993a. Los anticlinorios de Huizachal-Peregrina y de Huayacocotla: dos partes de la Fosa de Huayacocotla-El Alamar. I. El Alogrupo Los San Pedros. Boletín de la Asociación Mexicana de Geólogos Petroleros, 43, 1-33.

Rueda, G. J., Dueñas, M. A., Rodríguez, J. L., Minero, M. y Uribe, G. 1993b. Los anticlinorios de Huizachal-Peregri- 
na y de Huayacocotla: dos partes de la Fosa de Huayacocotla-El Alamar. II. Bioestratigrafía, Cronoestratigrafía y Paleoecología del Alogrupo Los San Pedros. Boletín de la Asociación Mexicana de Geólogos Petroleros, 43(2), 1-29.

Ruiz, J., Patchett, P. J. and Ortega-Gutierrez, F. 1988. Proterozoic and Phanerozoic basement terranes of Mexico and Nd isotopic studies. Geological Society of America Bulletin, 100, 274-281.

Sour-Tovar, F., Quiroz-Barroso, S. A. and Navarro-Santillán, D. 1996. Carboniferous invertebrates from Oaxaca, MidContinent paleogeographical extension. 1996 Geological Society of America Annual Meeting. Denver Colorado. Abstracts with Programs, p. A-365.

Sour-Tovar, F., Álvarez, F. and Martínez Chacón, M.L. (en prensa). Lower Mississippian (Osagean) spire bearing brachiopods from Cañón de la Peregrina, North of Ciudad Victoria, Tamaulipas, northeastern Mexico. Journal of Paleontology.

Stewart, J.H, Blodgett, R.B., Boucot, A.J. and Carter, J.L. 1993. Middle Paleozoic terrane near Ciudad Victoria, northeastern Mexico, and the southern margin of Paleozoic North America. In: Proceedings of the First Circum-
Pacific and Circum-Atlantic Terrane Conference. Guanajuato (Eds. F. Ortega-Gutiérrez, P. J. Coney, E. CentenoGarcía, and A. Gómez-Caballero). Instituto de Geología, Universidad Nacional Autónoma de México, 147-149.

Stewart, J.H, Blodgett, R.B., Boucot, A.J., Carter, J.L. and López, R. 1999. Exotic Paleozoic strata of Gondwanan provenance near Ciudad Victoria, Tamaulipas, México. In: Laurentia-Gondwana connections before Pangea (Eds. V.A. Ramos and J.D. Keppie). Geological Society of America, Special Paper 336, 227-252.

Sturgeon, M.T. and Hoare, R.D. 1968. Pennsylvanian brachiopods of Ohio. Ohio Geological Survey Bulletin, 63, 95 pp.

Williams, A., and Brunton, C. H. C. 1997. Morphological and anatomical terms applied to brachiopods. In: Treatise on Invertebrate Paleontology. Part H. Brachiopoda (Revised) 1 (Ed. R. Kaesler). New York and Lawrence, Geological Society of America and University of Kansas Press, 350-362.

Manuscrito recibido: 9 de Mayo, 2003 Manuscrito aceptado: 28 de Mayo, 2004 